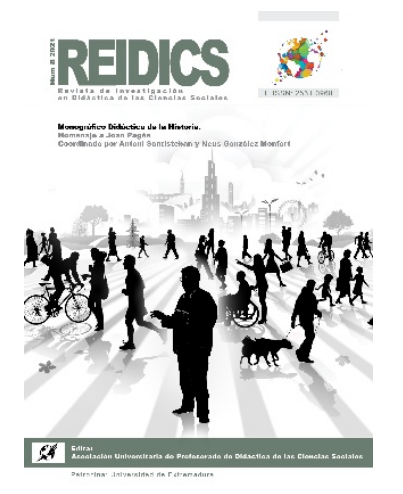

\title{
REIDICS
}

Revista de Investigación en

Didáctica de las Ciencias

Sociales
Núm. 8, 2021

Recibido 2 de febrero de 2021

Aceptado 15 de marzo 2021

E-ISSN: 2531-0968

\section{¿Hacia dónde caminan los cangrejos? Perspectivas de futuro de una generación asestada por dos crisis ${ }^{1}$}

\author{
Where do crabs walk? Future prospects of a generation plagued \\ by two crises
}

Rafael Olmos Vila
IES Bernat de Sarrià de Benidorm
Email: rafa.olmos@iesbernatdesarria.com
ORCID: https://orcid.org/0000-0001-7229-1538
DOI: https://doi.org/10.17398/2531-0968.08.106

\section{Resumen}

La generación de jóvenes actuales se ha enfrentado a dos crisis relativamente cercanas, que condicionan sus expectativas de vida y posibilidad de alcanzarlas. La investigación indaga sobre la evolución de las representaciones sociales de un mismo grupo de 23 chicas y 10 chicos sobre las crisis económica y sanitaria y sus perspectivas de futuro en dos contextos adversos. En un estudio longitudinal se comparan los resultados obtenidos en 2015 -siete años después del estallido de la crisis financiera, cuando se encontraban cursando Bachillerato- con sus representaciones este año 2020, durante la pandemia, cuando se encuentran realizando estudios superiores o trabajando. Se recoge la información a través de un cuestionario en línea sobre las causas, los responsables, las consecuencias, posibles soluciones y perspectivas de futuro. Los resultados muestran la continuidad de algunas ideas entre ambas crisis, como la escasa confianza en los gobernantes políticos, pero un cambio importante en la autopercepción sobre sus capacidades. En 2015 su confianza en poder cumplir sus proyectos vitales y laborales era plena, mostrándose muy optimistas y restando cualquier influencia de la crisis en poder culminarlos. Sin embargo, cinco años después, adaptan sus planes de futuro a la situación económica: ahora son más realistas, otorgando mayor incidencia a la crisis y al desempleo en sus vidas laborales. Reconocen las dificultades del contexto actual, considerando más de la mitad que vivirán peor que sus padres.

\footnotetext{
${ }^{1}$ En el año 2017 en las XIV Jornades Internacionals de Recerca en Didàctica de les Ciències Socials de la Universidad Autónoma de Barcelona, presentábamos junto a Joan una comunicación que portaba por título "Quines són les aspiracions socioprofessionals i els projectes de vida dels nois i les noies en un context de crisi econòmica?" Qué piensan los y las adolescentes, cómo sienten, cómo ven el mundo y cómo pueden transformarlo, siempre han sido finalidades de sus investigaciones. Ahora conviene preguntarse cómo aquellos proyectos de vida ideados durante la crisis económica, se han visto afectados durante la pandemia. Sirva esta continuidad de humilde homenaje y aprecio por todo su magisterio y huella en mí.
} 
Palabras clave: representaciones sociales; crisis económicas; crisis covid-19; imágenes del futuro; transiciones vitales.

\begin{abstract}
The current generation of young people has faced two relatively close crises, which condition their life expectations and the possibility of reaching them. The research looks into the evolution of the social representations of a same group of 23 girls and 10 boys on the economic and health crisis and their future prospects in two difficult contexts. In a longitudinal study, the results obtained in 2015 -seven years after the outbreak of the financial crisis, when they were in high school- are compared with their representations this year 2020, during the pandemic, when they are pursuing higher education or working. Information is collected through an online questionnaire about the causes, the people responsible, the consequences, possible solutions and future prospects of the crisis. The results show the continuity of some ideas through both crises, such as low confidence in political leaders, but an important change in self-perception of their capabilities. In 2015, their confidence in fulfilling their vital and work projects was full, showing themselves very optimistic and subtracting any influence of the crisis in completing them. However, five years later, they are adapting their future plans to the economic situation, being realistic, giving greater incidence to the crisis and unemployment in their working lives. They recognize the difficulties of the current context, considering more than half of the group parents did.
\end{abstract}

Keywords: social representations; economic crisis; covid-19 crisis; images of the future; life transitions.

\title{
1. Introducción
}

Los medios de comunicación han bautizado a la generación actual de jóvenes como los cangrejos (Morán, 2012), por dar socioeconómicamente pasos hacia atrás respecto a sus progenitores; también como la generación boomerang por no poder mantener su independencia, sus viviendas, y volver a sus casas (Donat y Martín-Lagos, 2020; Vallejo, 2017). En ambas etiquetas destaca su carácter regresivo. Supuestamente se trataría de la primera cohorte generacional desde la Segunda Guerra Mundial que vivirá peor que sus ascendientes, rompiéndose así la dinámica del ascensor social, por la cual las generaciones futuras recibían un legado que las aupaba a los hombros de sus progenitores. La idea de la movilidad intergeneracional ascendente, la capacidad de progresar mediante la formación, el mérito y el esfuerzo, partiendo de un contexto de igualdad de oportunidades, se presenta como una de las bases de las democracias y del contrato social (Sandel, 2020). Sin embargo, las investigaciones en España (Gil-Hernández, Bernardi y Luijkx, 2020) y el resto del mundo (Breen, 2019; Chetty, Grusky, Hendren, Hell, Manduca y Narang, 2017) muestran que el progreso social entre las generaciones no ha sido una constante lineal y, en general, a partir de los años ochenta, se invierte la tendencia hasta la actualidad, llegando a encontrar los cangrejos más dificultades que sus padres para construir un proyecto vital estable (Barreiro, 2017; Estefanía, 2017; Politikon, 2017).

Más allá de los datos económicos, conviene preocuparnos por la generación actual, doblemente golpeada en sus sueños (Aumaitre y Galindo, 2020), y preguntarnos si sigue confiando en el discurso providencialista de las democracias liberales o si por el contrario ha asumido el pesimismo. 
En sus cortas vidas ya han vivido dos crisis relativamente cercanas, la financiera en 2008, con gran destrucción del empleo, polarización social, prolongada en el tiempo y de lenta recuperación, hasta conectarse con la actual crisis de la covid-19: "Y como consecuencia de todo ello, la quiebra de sus expectativas de futuro, materiales o emocionales, que es la herida más lacerante de estos años bárbaros" (Estefanía, 2017, p.661).

La actual coyuntura de emergencia sanitaria y de infoxicación de información (Olmos, 2020) añade, a la incertidumbre heredada, el temor. La vulnerabilidad emocional del contexto hace más difícil construir un plan de futuro, trazar un juicio crítico sobre los acontecimientos (Castellví, Massip y Pagès, 2019) y a la vez socava su confianza en el futuro.

\section{Marco teórico: ¿Qué piensan los jóvenes sobre los problemas que les afectan?}

\subsection{Los jóvenes ante la crisis de 2008}

La crisis de 2008 despertó diferentes movimientos sociales en los cuales los jóvenes tuvieron un gran protagonismo activo (Freixa y Nofre, 2013). Los milenials no encontraron -ni encuentran- soluciones en la política tradicional, solo un empeoramiento de su situación (Olmos, 2016; Pagès, 2015); en consecuencia, se observa un giro en sus decisiones políticas y la necesidad de construir un nuevo discurso:

En España, la quiebra del bipartidismo no se habría producido de no ser por los jóvenes. Según la encuesta poselectoral del CIS para las elecciones de junio de 2015, únicamente el 21 por ciento de los jóvenes entre 18 y 32 años vota al PP y al PSOE, mientras que el 50,3 por ciento de ellos lo hace por uno de los dos partidos emergentes (el 34 por ciento por Podemos y el 16,5 por ciento por Ciudadanos). Si echamos la vista atrás en el tiempo, se descubre que en anteriores comicios los jóvenes no presentaban ninguna pauta diferenciada: votaban al bipartidismo en la misma medida que otras generaciones. (Barreiro, 2017)

Hay un crecimiento de la movilización: participación en huelgas, manifestaciones, interés por las cuestiones políticas y económicas... pero ¿se ha conseguido la alfabetización financiera de la sociedad? ¿La ciudadanía y los adolescentes son críticos con el sistema financiero? ¿Exigen el control político y responsabilidades de los mercados para evitar otra crisis?

Las investigaciones durante estos años han sido impulsadas por la Association for European Economic Education, a través de la revista Journal of Social Science Education y el Handbook de investigación sobre educación financiera (Aprea, Wuttke, Bruer, Koh, Davies, Greimel-Fuhrmann y Lopus, 2016). Más de una década después del estallido de la crisis económica, podemos presentar cuál es la interpretación de los adolescentes de las causas de la crisis, la responsabilidad de los diferentes actores, las posibles soluciones y las consecuencias que creen implica en sus futuros.

En una investigación desarrollada en Alemania en 2009, Aprea y Sappa (2014) entrevistaron a 56 adolescentes entre 14 y 19 años. Solo un 5\% negaron la crisis económica, atribuyendo su existencia a una invención de los poderes fácticos para generar miedo, controlar 
a la población y obtener mayor beneficio económico. La mitad de los 56 jóvenes, sí que reconocía la existencia de la crisis, pero solo la explicaba desde un enfoque monocausal, destacando el desempleo como el principal síntoma y exponiendo, pero sin razonar, las soluciones más presentes en los medios de comunicación: incentivar el consumo desde el gasto público o subvencionar la compra de vehículos. Sus relatos de la crisis no conseguían explicar su progresión desde las causas hasta las consecuencias. Entendían la crisis como un fenómeno coyuntural, que sería breve en el tiempo y que no les afectaría individualmente. Otro 30\% si fue capaz de presentar una narración elaborada de la crisis, pero en su evaluación del impacto de la crisis, excluía a su círculo cercano de sufrir las consecuencias. Solo un $15 \%$ se mostró más realista, reconociendo que podría tener influencia en sus futuros personales.

Las mismas cuestiones se preguntaron a jóvenes de Italia y Suiza (Berti et al., 2017). Sus explicaciones sobre la crisis fueron simplistas: solo el 40\% fue capaz de nombrar más de dos causas, la mayoría sólo citándolas y sin conectarlas en una explicación coherente. Mientras que los ítalos destacaron como la principal causa la ineptitud de los políticos, restando responsabilidad a los bancos, los adolescentes suizos, sí les señalaron como culpables. La confianza de ambos grupos de estudiantes en sus futuros laborales se mostró firme: solo 1 de cada 10 , tenía incertidumbre ante la incidencia de los acontecimientos.

Mientras que en las entrevistas de Aprea y Sappa (2014) se preguntaba a los adolescentes qué medidas estaba implementando el gobierno, en otra investigación se interpelaba a los adolescentes a proponer soluciones (Olmos, González-Monfort y Pagès, 2017). Una población de 68 alumnos, entre 16 y 18 años, aportaban soluciones a los problemas reflejados en cuatro noticias de prensa sobre los desahucios, el desempleo, las preferentes y el éxodo universitario. En torno al 50\% no supo exponer una medida al problema económico y social que se les presentaba, pero se mostraron muy optimistas: el $95 \%$ evaluaba que la crisis no afectaría sus planes futuros. Un optimismo similar al de otras investigaciones desarrolladas con adolescentes durante la crisis en España (Anguera, 2012). Paradójicamente la noticia del éxodo universitario, el problema de las noticias que generacionalmente debía resultarles más cercano, fue el que registró mayor porcentaje de respuestas en blanco (62\%).

La debilidad de sus argumentaciones sobre la crisis y la ingenuidad de sus propuestas de solución evidencia el déficit de su formación en educación financiera y economía general. En los primeros trabajos de Delval y Denegri (2002) sobre el sistema bancario, los estudiantes ya proponían la máquina de hacer dinero como solución, y, casi una década después, en investigaciones sobre la crisis financiera de 2008 (Delval y Kohen, 2010), volvía a ser una de las soluciones formuladas, sin considerar los costes inflacionarios en un contexto de endeudamiento. Parece que aquellos que no son capaces de explicar y comprender los acontecimientos económicos, ni simplemente las causas del pasado, confían mágicamente en la intervención de la providencia en sus futuros: "In a kind of "magical thinking," they argued that things will turn out positively in the end, without having a clear idea of how this could be the case" (Aprea y Sappa, 2014, p.62).

Este optimismo inocente en el futuro, que les hace inmunes al impacto de la crisis, da un giro cuando llegan al final de sus periodos de formación académica o han tenido sus primeras 
experiencias laborales. A pesar de tener mejor o peor conocimiento financiero de la crisis económica, su perspectiva sobre el futuro es más negativa: el contacto con la realidad laboral baja sus expectativas a la tierra. Un estudio realizado por la empresa demoscópica MyWord, (Barreiro, 2018) sobre una población de más de 600 jóvenes de 18 a 35 años mostraba su pesimismo en el futuro: 6 de cada 10 creen que vivirán peor que sus padres, vislumbran un retroceso por el desempleo, los trabajos precarios y las dificultades de emanciparse. Más del $90 \%$ de los entrevistados responsabiliza a los gobernantes políticos de esta situación, independiente de su grupo de edad: 18-37 años (93,8\%), 38-52 (94,9\%), 53-75 (92,3\%) (Barreiro, 2018, p.30).

Durante la transición del instituto o universidad hacia el mercado laboral se produce una fractura en su percepción sobre el futuro. Alonso, Fernández e Ibáñez (2016) realizaron dos grupos focales de jóvenes: el primero formado por trabajadores de la hostelería de 20-30 años, sin estudios o la secundaria obligatoria; y un segundo grupo constituido por becarios y trabajadores de 25-35 años, en formación de prácticas en empresas o universidades, cuya formación mínima era una licenciatura o ingeniería. Ambos, a pesar de la polarización académica, coincidían en asumir la precariedad de las condiciones laborales, la temporalidad y, en general, en dejar de confiar en el esfuerzo personal y, en menor medida, en la meritocracia.

El desengaño en la formación universitaria afloraba en los testimonios de la investigación de Zurdo y López de la Nieta (2013):

(...) el caso de los universitarios ubicados ahora al final de su juventud (en general de origen humilde), a los que se les 'prometió' socialmente que las credenciales educativas les garantizaban el acceso a un status socioeconómico medio/alto, y una profesión prestigiosa (es paradigmático el caso de los ingenieros superiores), pero a los que la crisis bloqueó el acceso al mercado laboral. Se identifica con posiciones del eterno "becario" que sufre humillaciones y es explotado, expresando una máxima disonancia entre el desempeño de labores hipercualificadas y condiciones laborales vergonzosas. Este colectivo se encuentra en un verdadero estado de shock: la capacidad y el esfuerzo no han servido para nada, pero ya no es posible cambiar de estrategia. Insisten obsesivamente en la vía meritocrática, pero, al mismo tiempo, son los que elaboran una crítica más demoledora acerca de la degradación y corrupción del modelo meritocrático. Identifican una meritocracia infame, falsa y vil que no ha correspondido a sus esfuerzos, sacrificios y a los méritos acumulados: "hemos sacrificado muchas cosas para conseguir esta carrera [ingeniería superior]", "estudiando como un monje desde los dieciocho años". (p.408-409)

\subsection{Los jóvenes ante la crisis de $\mathbf{2 0 2 0}$}

El contexto de la pandemia y especialmente los meses de confinamiento, han condicionado la metodología de muchas de las investigaciones, realizándose a partir de cuestionarios en línea.

Los efectos inmediatos en la formación y situación laboral de los jóvenes son traumáticos (Eurofound, 2020). Así lo refleja la encuesta mundial a más de 12.000 jóvenes de 18 a 29 años de 112 países, realizada entre abril y mayo: 1 de cada 6 jóvenes había dejado de trabajar desde el inicio de la pandemia y el $45 \%$ había visto disminuir sus ingresos al reducir su jornada laboral. Un escenario de mayor precarización que dificultará y ralentizará la transición al mercado laboral de esta generación (OIT, 2020). El mismo informe pone de relieve los efectos en el ánimo: el 38 por 
ciento de los jóvenes, a nivel mundial, se sienten inseguros ante sus futuras perspectivas profesionales y el 16 por ciento siente temor al respecto (p.36).

En Italia, el país más afectado por la pandemia en sus principios, se desarrolló en los meses de abril-mayo una investigación para conocer la dinámica psicosocial de la población durante el confinamiento (Barbieri, Compare, Guarino, Tzankova y Albanesi, 2020). Al ser preguntados por las implicaciones de la crisis en el futuro, la población encuestada señaló un empeoramiento de su situación económica respecto a la anterior a la covid-19. El grupo de 18-21 años fue el segundo más pesimista en su futuro.

Al otro lado del océano, el estudio del Instituto Archbridge a 2223 encuestados en los meses de julio y agosto (Schwarz, 2020) mostró la confianza de los estadounidenses en la permanencia y viabilidad en cumplir el sueño americano. El 18\% de los jóvenes de 18 a 29 años consideraban ya haberlo alcanzado; el $48 \%$ eran optimistas al valorar que estaban en el camino para lograrlo; mientras que el 31\% lo situaban fuera de su alcance. La encuesta se desarrolló mientras EEUU se convertía en el país con más muertos y casos confirmados por COVID-19 y en medio de protestas del movimiento Black Lives Matter, por la desigualdad racial y la brutalidad policial. A pesar de este contexto, el 36\% de los jóvenes creen que gozan de más oportunidades que sus progenitores, frente a un 19\% que valora encontrarse con menos posibilidades que sus ascendientes. Incluso la población de origen hispano o negra, en estas circunstancias adversas, confía en la movilidad intergeneracional ascendente:

As the survey shows, for the majority of Americans (even in the midst of challenging times) the American Dream is alive and well, the vast majority of survey respondents reported that they have either achieved or are on their way to achieving the American Dream. (...) Large majorities, and especially black Americans, believe that they have more opportunities than their parents had and that their children will have either the same number or more opportunities for success as they did (p.10).

En el caso de los jóvenes españoles, encontramos una percepción sobre sus futuros similar a otros jóvenes europeos. Los índices económicos del verano, a pesar de reactivarse la economía después del confinamiento, han empeorado los datos estructurales de trabajo: solo tienen empleo el 33,5\% de los jóvenes (16-29 años), incluyendo los que se encuentran en ERTE. En verano las contrataciones de jóvenes descendieron un 14\% y casi un 30\% de la población ocupada corre riesgo de perder sus empleos (INJUVE, 2020).

Hay que destacar la progresión de los sentimientos pesimistas hacia el futuro. El estudio de las series temporales de las encuestas del Centro Reina Sofía, permite conocer la evolución de la confianza de los jóvenes en el futuro, desde la crisis inmobiliaria hasta ahora:

Ante la pregunta ¿qué crees que pasará en los próximos años? En 2015 nos respondía hasta un $74 \%$ que creía muy o bastante probable tener que trabajar en lo que fuera y un $67 \%$ que tendría que depender económicamente de su familia (Megías y Ballesteros, 2016). En 2019 parecía que las expectativas remontaban y esos porcentajes se reducían al 58\% y $36 \%$ respectivamente, pero es evidente que la nueva crisis sociosanitaria ha vuelto a impactar sobre las trayectorias juveniles. De hecho, entre marzo y mayo de 2020 ya detectamos cómo se incrementan las sensaciones de incertidumbre, de ansiedad y de desánimo, mientras que disminuyen en gran medida las sensaciones de curiosidad y entusiasmo ante el futuro. (Sanmartín, Ballesteros, Calderón y Kuric, 2020, p.76) 
En estos años de juventud no solo se forman académicamente o realizan las prácticas para incorporarse al mundo laboral, sino que también se construyen los sueños, ilusiones y frustraciones, así como su confianza y desánimo en la política, por este motivo

el estudio de las emociones es relevante en el campo de la didáctica de las ciencias sociales ya que las emociones juegan un papel determinante en el aprendizaje de los contenidos históricos, sociales y geográficos, y son un elemento fundamental en la toma de decisiones de la ciudadanía (Castellví, Massip y Pagès, 2020, p.24-25)

\section{Metodología de la investigación}

\subsection{Tipo de investigación}

La investigación es un estudio longitudinal sobre los cambios y continuidades en las opiniones, creencias y perspectivas de futuro de un mismo grupo de 33 adolescentes durante las dos crisis: económica, cuando estudiaban en Bachillerato (2015), y sobre la crisis actual de la COVID-19, cuando la mayoría se encuentran en el ecuador de sus grados universitarios.

Siguiendo la teoría del ciclo vital, life span theory, (Heckhausen, Wrosch y Schulz, 2010), entendemos que la finalización del Bachillerato y cursar un grado de FP o universitario, es una etapa clave en la transición hacia al mercado laboral por las implicaciones que tiene en sus proyectos de vida. Sin embargo, el arquetipo de transición hacia la adultez, lineal y jalonado (terminar los estudios, empezar un trabajo, emanciparse del nido familiar, formalizar una relación de pareja y asumir la paternidad/maternidad), no se ajusta a la realidad: ni es lineal, ni exige cumplir todas las metas (Sánchez-Galán, 2020). El modelo de transición es más bien un vector de doble sentido, las llamadas transiciones yo-yo (Du Bois-Reymond y López Blasco, 2004). En las generaciones boomerang que estudiamos, se observa perfectamente en el hecho de que pierdan la emancipación residencial volviendo al hogar familiar.

Las crisis son momentos que tensionan, aún más, los procesos de transición y la dialéctica entre las decisiones y deseos individuales frente al peso de los condicionantes estructurales e institucionales (Furlong, Woodman y Wyn, 2011; Tarabini, Curran, Castejón y Montes, 2018). Los cambios continuos en las vidas de los individuos y su inestabilidad nos obligan a realizar un estudio longitudinal para tener una perspectiva sólida. Además, algunos autores rechazan etiquetar a las generaciones, ya que supone homogeneizar sueños, inquietudes y dificultades a toda una cohorte, por este motivo abogan por realizar estudios longitudinales e individuales (Rudolph y Zacher, 2020).

\subsection{Contexto}

Los jóvenes de nuestra investigación realizaron, durante los años de la crisis financiera, su educación primaria y secundaria en Benidorm. Actualmente el 85\% sigue viviendo en Benidorm, cursando la mayoría estudios en la Universidad de Alicante, viviendo el resto en la ciudad de Alicante (6\%), Valencia (3\%) o en otra comunidad (6\%). Benidorm es una ciudad mediterránea que fundamenta su economía en el turismo y el sector servicios. Durante estas dos crisis se ha 
evidenciado aún más la dependencia del turismo: en verano se duplica el número de contrataciones, pero se destruyen con el inicio del otoño, confirmando el modelo económico de sol y playa. La reforma de 2012 agravó la precarización con cambios en la regulación de la negociación colectiva y facilitando modificaciones unilaterales en las condiciones laborales y la externalización de muchos trabajos vinculados con la hostelería (Beltrán y Pino, 2017). Durante la pandemia, las prohibiciones de vuelos internacionales y los decretos del gobierno, restringiendo las actividades comerciales y lúdicas (Serrano, Soler y Pascual, 2020) han paralizado la economía y aumentado el desempleo.

\subsection{Preguntas y objetivos}

Las preguntas que nos formulamos son las mismas que nos planteamos en la investigación de 2015 (Olmos y Pagès, 2018), pero adaptadas a la nueva coyuntura. Entonces, en 2015, nos preguntamos:

¿Cómo ven los adolescentes su futuro laboral? ¿Y social y colectivo?

¿Cuáles son las causas, los responsables y las consecuencias de la crisis económica? ¿En quién depositan la confianza para encontrar soluciones a la crisis económica?

Ahora, la distancia en el tiempo nos permite comprobar el grado de consecución de sus proyectos y su optimismo en poder culminarlos:

¿Han cambiado sus proyectos de vida, las rutas profesionales que se marcaron? ¿Se ha debilitado o reforzado su confianza en alcanzarlos?

¿Creen que vivirán mejor que sus padres y madres?

¿Cuáles son las causas, los responsables, las consecuencias y posibles soluciones de esta crisis?

El objetivo principal es indagar sobre sus proyecciones de futuro, para valorar si el contexto pandémico y la etapa de educación superior -esta nueva transición hacia el mercado laboral, tener las primeras experiencias (contratos de verano, minijobs, combinar estudios y trabajo...)- reducen o potencian la convicción y aspiraciones de años atrás.

En segundo lugar, averiguar e interpretar sus opiniones sobre los principales actores de la crisis sanitaria, las causas, consecuencias y soluciones. Profundizar cómo permanecen o cambian del escenario económico al de la salud, entendiendo los contextos en los cuales se conforman.

\subsection{Muestra}

La muestra estuvo integrada en Bachillerato por 35 adolescentes de edades entre 16 y 18 años. Pudimos contactar con 33 (23 chicas y 10 chicos, de 20-22 años), de modo que tomamos los testimonios de estos 33 adolescentes a finales de 2015 y ahora, para poder realizar la reconstrucción de sus recorridos vitales e ideas. 


\subsection{Instrumentos}

Los instrumentos utilizados han estado condicionados por las limitaciones de movilidad de la pandemia. Al igual que las investigaciones del marco teórico hemos optado por una encuesta en línea, a través de la herramienta de formularios google.forms.

Las preguntas mixtas contemplaban varias opciones y siempre "otra", pudiendo especificarla:

¿Tu situación laboral/académica es?

¿Te ves en el futuro trabajando de lo que estudias?

¿De qué te gustaría trabajar? Ofrecimos una lista formada por las profesiones que eligieron en 2015, añadiendo la opción otra.

¿Qué es lo que más valoras de un trabajo?

¿Quién o qué consideras responsable de la crisis de la covid-19?

¿Cuál crees que es la principal consecuencia de esta crisis?

¿Quién o qué consideras capaz de solucionarla?

¿Crees que en el futuro tu situación económica será mejor, peor o igual que la de tus padres?

\subsection{Temporalización}

La información se recopiló por primera vez durante la segunda semana de diciembre de 2015, días antes de las elecciones generales del 20D. En aquel contexto los medios de comunicación y las campañas políticas de algunos partidos postulaban la recuperación económica, los brotes verdes, y haber pasado los años más duros desde el rescate financiero (Báñez, 2015). El CIS (2015) publicaba en su estudio de diciembre que el $41 \%$ consideraba que la economía española mejoraría en los próximos seis meses, frente quienes valoraban que empeoraría (17\%).

En esta ocasión, el formulario en línea se envió la última semana de mayo de 2020, cuando la Comunidad Valenciana se encontraba al final de la Fase I de la desescalada y a las puertas de entrar en la Fase II (BOE, 2020). El grueso de respuestas se recibió el mismo día 27 de mayo y la última el 8 de junio.

\section{Resultados}

Los 33 alumnos que rastreamos su evolución se encuentran actualmente cursando un grado universitario (67\%), estudiando y trabajando (15\%), estudiando un ciclo formativo (9\%), trabajando (6\%) y en el paro (3\%)

En 2015 instamos al alumnado a que eligiera los tres trabajos deseados por orden de preferencia, qué querían estudiar y qué dificultades pensaban que iban a encontrar. Posteriormente, les preguntamos si la crisis que estábamos viviendo sería un impedimento en lograrlo.

El $100 \%$ de los chicos confiaban plenamente en cumplir sus objetivos, mientras que las chicas se mostraban más prudentes y realistas, al señalar que deberían realizar sacrificios, 
posiblemente marchar al extranjero, estar años de prácticas, trabajar para otros... pero el 95\% pensaba que lo lograría, aunque, a diferencia de los chicos, la crisis retrasaría su consecución.

En la actualidad los resultados son muy diferentes al examinar la incidencia de la crisis en sus futuros: sí, seguro, la crisis no influye (36\%); la actual crisis condiciona mi futuro, pero sí conseguiré trabajar de mi elección (33\%); no conseguiré trabajar de lo que estoy estudiando por la crisis (22\%); creo que seguiré manteniendo mi trabajo (6\%), seguiré en el paro (3\%). La proporción de chicos y chicas en cada apartado fue similar, no observamos diferencias relevantes.

Cabe recordar que la mitad de los chicos elegía, en 2015, futbolista como primera opción, y en ocasiones era la única que aparecía. Solo algunos situaban, en segundo lugar, entrenador o fisioterapeuta, pero siempre vinculados al mundo del fútbol. Actualmente ninguno juega al fútbol de manera federada.

Entre las prioridades que destacan en la elección de un trabajo, pudiendo elegir varias a la vez, priorizaron que les guste (90\%); el ambiente de trabajo (40\%); ganar suficiente dinero para vivir (35\%); ayudar a los otros (30\%); ganar muchísimo dinero (18\%) y estar cerca de casa (15\%).

Como podemos ver en la Tabla I, en 2015 la profesión mayoritaria entre las chicas era psicóloga (8), seguida de profesora de infantil (6), inglés (3) y educación especial (1), destacando que les gustaba, era su vocación y querían ayudar a los demás. Actualmente solo 1 alumna cursa psicología, mientras que los 5 futbolistas estudian economía (4) y fisioterapeuta (1).

Tabla 1

Profesiones deseadas en 2015 y perspectiva en 2020

\begin{tabular}{|c|c|c|c|c|}
\hline & \multicolumn{2}{|c|}{ Chicos } & \multicolumn{2}{|c|}{ Chicas } \\
\hline & 2015 & 2020 & 2015 & 2020 \\
\hline Futbolista & 5 & & & \\
\hline Economista & 1 & 4 & & \\
\hline Periodista & 1 & & & \\
\hline Geógrafo & 1 & 1 & & \\
\hline Taxista & 1 & & & \\
\hline Bombero & 1 & & & \\
\hline Fisioterapeuta & & 1 & & 1 \\
\hline Historiador/a & & 1 & & 1 \\
\hline Policía & & & 1 & 2 \\
\hline Psicóloga & & & 8 & 1 \\
\hline Profesora de infantil & & & 6 & 1 \\
\hline Profesora de primaria & & & 1 & 4 \\
\hline Profesora de educación especial & & & 1 & \\
\hline Profesora de inglés & & & 3 & 2 \\
\hline Traductora & & & & 1 \\
\hline Diseñadora de moda & & & 2 & \\
\hline Notaria & & & 1 & 1 \\
\hline Tatuadora & & & 1 & \\
\hline Trabajando en un comercio & & 2 & & 1 \\
\hline Parado & & 1 & & \\
\hline Guía turística & & & 1 & 1 \\
\hline Bióloga & & & & 1 \\
\hline Dibujante de cómics & & & & 2 \\
\hline Relaciones laborales recursos humanos & & & & 2 \\
\hline Obstetra & & & & 1 \\
\hline Bibliotecaria & & & & 1 \\
\hline
\end{tabular}

Fuente: Olmos (2018a) 
Respecto al papel de los protagonistas de la crisis, identificaban como culpables de la crisis económica a los políticos (85\%), pero en la pandemia, sólo lo es el gobierno por su mala gestión para el 21\%; nadie, ya que su origen es animal (37\%); China, por fabricarlo (33\%) y las élites (9\%), especificando algunos a Georges Soros y Bill Gates (Figura 1).

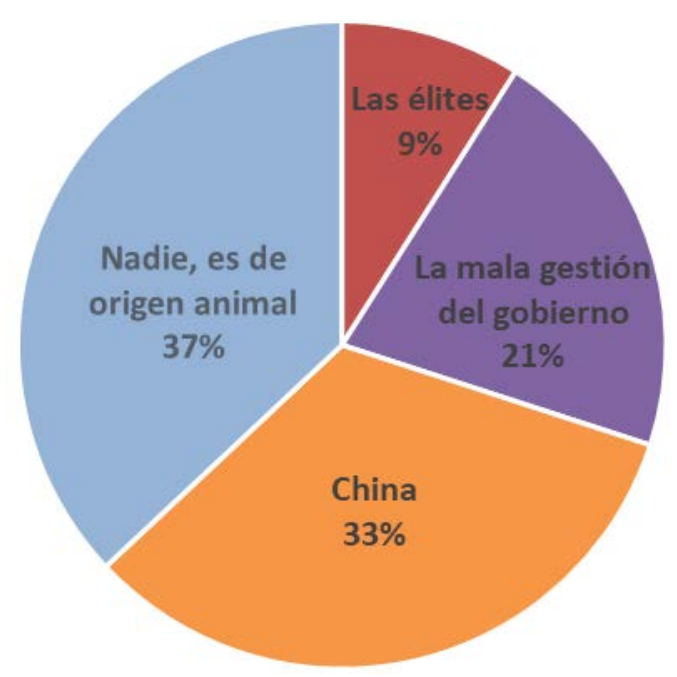

Figura 1. Responsables de la crisis actual

Fuente: Elaboración propia

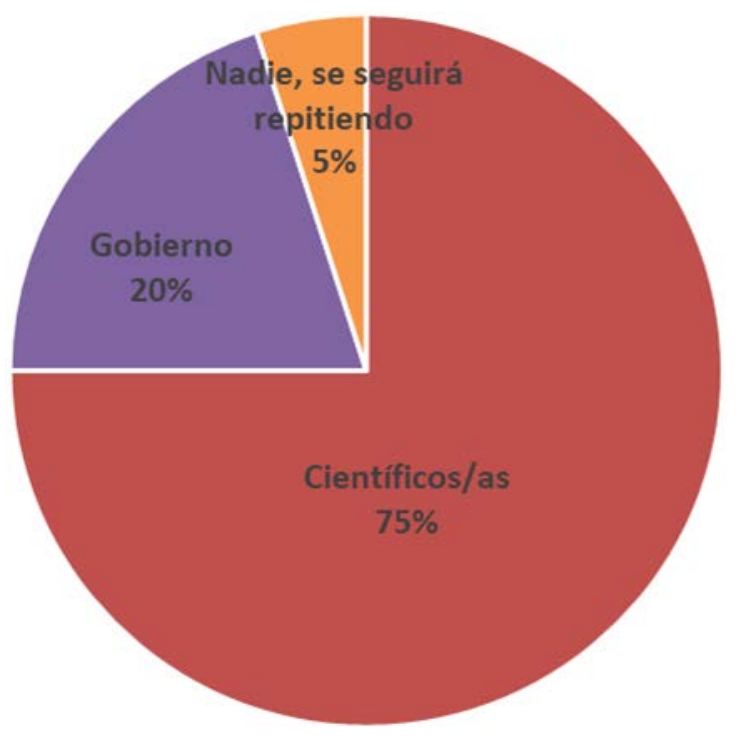

Figura 2. Confianza para salir de la crisis actual

Fuente: Elaboración propia

Las consecuencias antes y ahora que destacaron fueron eminentemente de tipo económico, vislumbrando un aumento del desempleo (85\%), pero en esta ocasión, también reconocían las 
implicaciones emocionales (12\%), destacando la depresión y la ansiedad, y demográficas por las defunciones (3\%).

Casi la mitad no supieron o no confiaban en nadie para superar la crisis económica, dejando en blanco su respuesta o especificando "nadie" (45\%). Su desencanto en la clase política les hacía mirar hacia la ciudadanía (15\%), en nuevos políticos renovadores (15\%), en políticos autoritarios del pasado (9\%) y en el gobierno de entonces (7\%). Ahora confían en los científicos (75\%), el gobierno (20\%) y en nadie, ya que "se seguirán repitiendo las pandemias" (5\%). La oposición fue una opción que ofrecimos, pero ninguno la seleccionó (Figura 2).

Sobre si son la generación cangrejo, en la Figura 3 podemos comprobar que para más de la mitad el futuro será negativo y creen que vivirán peor que sus padres (57\%), casi un cuarto cree que será mejor (24\%) y el resto que vivirán igual (19\%).

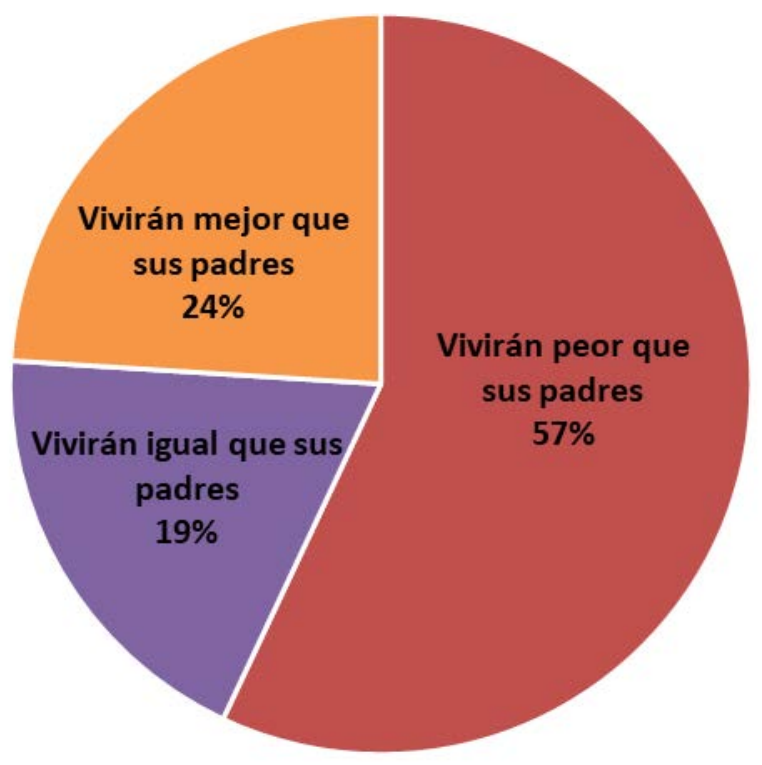

Figura 3. Confianza en vivir mejor que sus progenitores

Fuente: Elaboración propia

\section{Discusión}

La evolución de los planes laborales de nuestro alumnado, durante los años de la transición del IES a la Universidad o al mercado laboral, nos indica que se produce un desengaño respecto a sus propósitos iniciales. Los adolescentes empiezan a intuir la dureza de la realidad y a vincular sus perspectivas individuales a la marcha de la economía. Del exacerbado optimismo juvenil, que aislaba sus planes del contexto, "l'alumnat aspira, i creu que ho aconseguirà, a tenir un futur benestant, independent dels esdeveniments actuals del món" (Anguera, 2012, p.87) se pasa a la prudencia y cierta subordinación a las circunstancias coyunturales.

Las investigaciones sobre la convicción de estudiantes de secundaria o bachillerato en sus futuros individuales, aun ser realizadas durante la crisis (Anguera, 2012; Olmos, 2018b; Olmos y Pagès, 2017; Verdugo, Freire y Sánchez-Sandoval, 2018) muestran que no interfiere en sus deseos. 
Así nuestra población presentaba resultados optimistas similares en 2015, cuando cursaba Bachillerato. Sin embargo, en la siguiente década, cuando la franja de edad supera los 20 y en especial los 25, se invierten las estadísticas y sus relatos sobre el presente y el futuro son pesimistas (Alonso et al., 2016 y 2017; Zurdo y López de la Nieta, 2013). Esta involución de la confianza en cumplir sus sueños y aceptar el peso de la situación económica en sus vidas, que muestran jóvenes en paro, que han terminado sus estudios, trabajan de prácticas o trabajos precarios... la empezamos a observar en nuestro grupo de estudio, a pesar de no haber finalizado aún sus estudios. Las crisis, sí que han significado un golpe en su autopercepción de sus expectativas laborales, valorando con mayor cautela su porvenir. Sus ilusiones ingenuas transitan, en algunos casos, hacia sueños que se empiezan a frustrar o finiquitar como es el caso de los futbolistas.

También hay una transvaloración de los argumentos que les llevaron en 2015 a querer cursar sus estudios. Parece que asumen la competitividad, la escasa oferta de empleo, reconociendo, entre sus motivaciones, que el trabajo también es un modo de vida, de supervivencia. Realizar el trabajo que les guste y disfrutar con ello, sigue siendo una cuestión principal, pero pierde importancia la función altruista, ya no están tan presentes argumentaciones como "quiero ayudar a los demás", "se me da bien escuchar" o "me hace feliz colaborar y ayudar a los otros", que justificaban en su etapa de Bachillerato.

Siguiendo las series de Eurofound (2012 y 2014) sobre las condiciones de vida y trabajo, con el paso de los años hay una creciente pérdida de ilusión y para los jóvenes españoles queda en un segundo plano la satisfacción en sus empleos:

Pero si hay algo en lo que los jóvenes españoles contrastan especialmente con sus contrapartes europeos es en la satisfacción laboral, y es que los niveles de satisfacción con el trabajo son particularmente bajos en España. Mientras que en países como Austria, Dinamarca o Finlandia prácticamente la mitad de los jóvenes se muestra altamente satisfecho con el trabajo, en España son apenas un 21,8\% los jóvenes con esos niveles de satisfacción altos. Además, también hay una baja proporción de jóvenes en España altamente satisfechos con la situación económica y financiera $(11,2 \%)$. (INJUVE, 2016, p.295)

La dificultad de obtener un trabajo y aceptar las condiciones de precariedad son evidentes, observándose en la emigración de jóvenes altamente preparados desde la crisis de 2008 (Freixa y Rubio, 2017), y registrándose una tasa de paro juvenil que triplica a la media europea (INJUVE, 2020).

Ambas crisis, la económica y sanitaria, a pesar de tener consecuencias económicas similares en el empleo de los jóvenes, tienen un origen distinto, por este motivo las causas y responsabilidades son muy diferentes entre los resultados de 2015 y 2020, atribuyendo la primera al papel de los políticos, pero con escasa incumbencia en la segunda. No obstante, la escasa confianza en la clase política es una constante, apareciendo en las encuestas del CIS (2016 y 2020), como uno de los principales problemas. Así, en abril, un mes antes que nuestra investigación, el 78\% mostraba el aumento de su desconfianza en el gobierno en la gestión de la crisis, solo el $22 \%$ afirmaba haber mejorado su imagen sobre el gobierno tras las medidas adoptadas (40 dba, 2020a). Un porcentaje similar al 85\% de nuestra investigación que, siete años 
después de su estallido en 2008, seguía señalando a la clase política por la génesis y medidas tomadas sobre la crisis económica.

Se trata de un círculo en el que existe una correlación entre el optimismo en las perspectivas sobre el futuro individual y la situación económica del país, y, al mismo tiempo, de ésta con la confianza/responsabilidad en la clase política (INJUVE, 2016). Si vinculan la recuperación económica con la capacidad de los gobernantes, mientras exista una valoración negativa de la política, el pesimismo en el futuro de la sociedad e individual persistirá. Solo 1 de cada 4 jóvenes de 18 a 24 años piensa que en un año la situación económica será mejor (CIS, 2020), confiando en las actuaciones del gobierno (45\%), pero subordinándola "debiéndose atenerse a los que digan y propongan siempre los expertos" (89\%). Ambos porcentajes, la confianza en los políticos y científicos, son proporcionales a nuestros resultados obtenidos.

El estudio de noviembre de la agencia de investigación 40db (2020b), sobre una población de 2000 personas mayores de 18 años -casi el 80\% tenían más de 35 años- obtuvo los siguientes resultados en sus encuestas ante el siguiente enunciado: La crisis del coronavirus dejará... desigualdad económica (79\%) y desigualdad entre generaciones de jóvenes y padres (56,6\%). La población no joven, también es consciente del empeoramiento de las condiciones para los jóvenes respecto a su generación. En cambio, la población joven que piensa que vivirá peor que sus padres se mantiene entre el 57\% en nuestra investigación y el $60 \%$ ( 40 db, 2020b). Son datos alarmantes, por dos motivos: el primero por tratarse de jóvenes en periodo de formación o iniciando sus primeros trabajos que deberían partir de un gran optimismo, no de sueños rotos, y el segundo, porque las investigaciones muestran que el nivel socioeconómico familiar y el origen migrante influyen altamente en las posibilidades de cumplir las expectativas propuestas, incluso en las repeticiones de curso (Tarabini, Curran, Castejón y Montes, 2018), sin embargo, incluso observando hechos externos que les afectan, los y las estudiantes terminan culpabilizándose y no siendo capaces de buscar razones de su éxito o fracaso más allá de su individualidad (Sánchez-Rojo y Prieto, 2020, p.591). Un planteamiento que les sobrecarga de frustración, responsabilidad en sus decisiones, elecciones y consecuencias, relegando el éxito en sus capacidades y esfuerzo (Sandel, 2020), para exonerar a las instituciones, como cuando se nos justificaba la crisis de 2008 con "el mensaje de castigo hacia quienes «vivieron por encima de sus posibilidades». Los restos culturales de la austeridad obrera siguen imponiendo la autocrítica y exigiendo asumir una cierta responsabilidad en lo ocurrido." (Alonso et al., 2016, p.359)

Si debemos detenernos en sus imaginarios por su incidencia en sus futuros, la mayoría de los jóvenes, el $62 \%$, piensan que tras la pandemia empeora su situación, agravándose los problemas que arrastran (alto desempleo, precariedad, adquirir una vivienda...). Solo un $24 \%$ cree que no tendrá que depender de sus familias (Sanmartín et al., 2020), de modo que no termina de culminar la independencia que supone una transición plena, y se refuerza la idea de una generación boomerang.

Por último, cabe señalar que la crisis actual ha tenido un efecto igualador por debajo de la confianza de ambos géneros en el futuro, mostrando chicos y chicas mayor pesimismo en alcanzar sus proyectos laborales. Son los chicos quienes han moderado sus sueños. En Bachillerato los chicos no dudaban en poder conseguir sus metas, para ellos no cabía otra opción; mientras que 
las chicas, sí que contemplaban la influencia de la crisis económica en sus futuros a la par que su autoconfianza en lograrlo era menor (OCDE, 2015). Su autopercepción sobre sus competencias y destrezas explicaba la elección de sus carreras universitarias y la mayor presencia de las alumnas en el Bachillerato Humanístico que en el Científico, "la autovaloración que chicas y chicos hacen de su rendimiento en las materias escolares puede determinar que, en cursos posteriores, opten por un determinado itinerario educativo y profesional" (Padilla, García-Gómez y Suárez, 2010, p.500). Siguen existiendo estereotipos sexistas en torno a las ciencias que dificultan el acceso y deseo de las mujeres hacia estos estudios (UNESCO, 2019), cabe señalar que las Matemáticas, la FísicaQuímica y la Tecnología son "las materias en las que las alumnas hacen una valoración más negativa de su rendimiento, lo cual nos indica que este ámbito del conocimiento sigue estando "alejado" de ellas" (García-Gómez, Padilla y Suárez, 2009, p.1391).

\section{Conclusiones}

El número especial de INJUVE Jóvenes y generación 2020 publicado en 2015 (INJUVE, 2015) se abría con el editorial: ¿Horizonte 2020 esperanza o advertencia? Entonces, en el año 2015, realizamos la primera investigación y ahora en 2020, podemos afirmar que la segunda crisis ha dibujado un horizonte más pesimista, un salto en estos cinco años hacia la prudencia en la construcción de sus futuros, debilitando su optimismo. La advertencia, las consecuencias de la crisis económica de 2008, han contribuido, junto al pesimismo del contexto de la pandemia, a restar sus esperanzas.

Salir del IES, es salir de una burbuja. Llegar a la universidad, tener los primeros contactos con el mundo laboral o realizar las prácticas supone una dosis de realidad. Su confianza en cumplir sus proyectos se acerca a la de los jóvenes que ya trabajan, que sufren la precarización y estacionalidad del trabajo, a pesar de encontrarse aún realizando sus estudios universitarios. Esta aceleración en su imaginario negativo se inscribe en una generación formada entre dos crisis y receptora de mensajes aciagos.

Asumen la actual situación y su larga permanencia en el tiempo, al no confiar en los gobernantes políticos y vislumbrar que vivirán peor que sus padres. Las crisis no son solo una cicatriz anímica, sino que heredan un legado económico lastrado, que retrasará la consecución de sus metas y su transición a la vida adulta. Una brecha generacional que ya han interiorizado y que dificultará su calidad de vida, como ya han aceptado. Ante esta situación, Joan nos anima en nuestra tarea docente en un mundo globalizado donde "cada vez es más urgente y necesario educar a nuestros niños y a nuestras niñas, a nuestra juventud desde una perspectiva que les sitúe en el mundo" (Pagès, 2019, p.6) para empoderar a nuestros jóvenes en transformar su realidad, por pesimista que parezca:

(...) en el contexto de profunda crisis económica en el que nos encontramos -es necesario insistir y profundizar en que el presente es producto de las decisiones y acciones que se tomaron en el pasado y que, por lo tanto, el futuro que se está construyendo será el resultado de las decisiones y acciones que tomemos ahora (Pagès y González-Monfort, 2010, p.56). 


\section{Limitaciones}

Debemos tener presente la limitación de los resultados por el tamaño de la muestra, pero su valor por realizar el seguimiento de las mismas personas durante 5 años.

El lugar del estudio, Benidorm, por su modelo económico y su oferta laboral, también debe tenerse presente a la hora de valorar los resultados. Las peculiaridades de su economía local dificultan extrapolar los resultados al resto de España. Los trabajos vinculados a la hostelería y a los servicios son las actividades más afectadas por las restricciones gubernamentales, pero además están vinculados con la formación profesional, cuando la mayor parte de nuestra muestra cursa estudios universitarios y no encuentra tantas posibilidades de empleo en la localidad.

Igualmente ser conscientes de la coyuntura, el confinamiento es un periodo excepcional en nuestras vidas. Ante un momento inaudito e inesperado, las emociones de la población se han polarizado entre el desánimo y la esperanza, mientras la ansiedad por estar encerrado en casa ha convivido con el miedo por salir a la calle (40db, 2020a; Buccolo, Ferro y Mongili, 2020; Sanmartín et al., 2020) e incluso se observa una polarización ideológica. Hemos intentado que las investigaciones que citamos se hayan desarrollado en los mismos meses que la nuestra, ya que las perspectivas y los ánimos son diferentes si realizamos la investigación durante el pico de contagios y la economía paralizada, que cuando se dispensan las primeras vacunas. Este escenario no debe hacernos rechazar los resultados de las investigaciones desarrolladas durante los meses de confinamiento o posteriores, sino pensar que su residuo emocional y su impronta en el imaginario individual y colectivo, son una huella que supera a una generación en concreto para hacer mella en toda la sociedad.

\section{Referencias bibliográficas}

40db (2020a). Estudio sobre la crisis del coronavirus II (abril 2020). Recuperado de https://n9.cl/7w0kj

40db (2020b). Estudio sobre la crisis del coronavirus III (noviembre 2020). Recuperado de https://n9.cl/0irq

Alonso, L. E., Fernández, C. J. e Ibáñez, R. (2016). De la moral del sacrificio a la conciencia de la precariedad. Un análisis cualitativo de los discursos sobre la evolución de la crisis en $\begin{array}{lllll}\text { España. } & \text { Política } & y & \text { Sociedad, } & \text { 53(2), }\end{array}$ https://doi.org/10.5209/rev_POSO.2016.v53.n2.49380

Alonso, L. E., Fernández, C. J. e Ibáñez, R. (2017). Juventud y percepciones de la crisis: precarización laboral, clases medias y nueva política. Empiria, 37, 155-178. https://doi.org/10.5944/empiria.37.2017.18983

Anguera, C. (2012). El concepte de futur en l'ensenyament de les ciències socials. Estudis de cas a l'educació secundària (Tesis doctoral). Universidad Autónoma de Barcelona.

Aprea, C. y Sappa, V. (2014). Variations of Young Germans Informal Conceptions of Financial and Economic Crises Phenomena. Journal of Social Science, 13(3), 57-67. https://doi.org/10.4119/jsse-710 
Aprea, C., Wuttke, E., Bruer, K., Koh, N.K., Davies, P., Greimel-Fuhrmann, B. y Lopus, J.S. (Eds.). (2016). International Handbook of Financial Literacy. Singapur: Springer.

Aumaitre, A. y Galindo, J. (2020). La generación de la doble crisis. Inseguridad económica y actitudes políticas en el sur de Europa. ESADE y FNS. Recuperado de http://itemsweb.esade.edu/research/EsadeEcPol_FNF_Generacion_Doble_Crisis.pdf

Báñez, M.F. (2015). Intervención de la Ministra de Empleo y Seguridad Social a la apelación del grupo parlamentario socialista, sobre el balance de la política de empleo en la X Legislatura. Diario de Sesiones del Congreso de los Diputados, Madrid, 30 de septiembre de 2015, Núm. 308, pp.1-57.

Barbieri, I., Compare, Ch., Guarino, A., Tzankova, I. y Albanesi, C. (2020). La comunità durante il lockdown: Report preliminare di ricerca. Bologna: Dipartimento di Psicologia, p. 51. http://doi.org/10.6092/unibo/amsacta/6528

Barreiro, B. (2017). La sociedad que seremos: Digitales, analógicos, acomodados y empobrecidos. Barcelona: Planeta.

Barreiro, B. (2018). Las perspectivas vitales de los jóvenes. Bruselas: Fundación Europea para los Estudios Progresistas. Recuperado de https://n9.cl/c5xhn

Beltrán, R. y Pina, S. (2017). Emprender desde la sororidad: las Kellys de Benidorm y las Aparadoras de Elche. En F. J. Cano-Montero y R. Calvo (coord.), Ciencias laborales y emprendimiento: discursos, retos y experiencias de aula (pp. 213-226) Valencia: Neopàtria.

Berti, A.E., Ajello, A.M., Aprea, C., Castelli, I., Lombardi, E., Marchetti, A., ... Valle, A. (2017). Adolescents' and Young Adults' Naïve Understandings of the Economic Crisis. Europe's Journal of Psychology, 13(1), 143-161. https://doi.org/10.5964/ejop.v13i1.1187

BOE (2020). Orden SND/414/2020, de 16 de mayo, para la flexibilización de determinadas restricciones de ámbito nacional establecidas tras la declaración del estado de alarma en aplicación de la fase 2 del Plan para la transición hacia una nueva normalidad. Boletín Oficial del Estado. Madrid, 16 de mayo de 2020, núm.138, 33312-33334.

Breen, R. (2019). Education and intergenerational social mobility in the US and four European countries. Oxford Review of Economic Policy, 35(3), 445-466.

Buccolo, M., Ferro, V. y Mongili, S. (2020). Percezioni e vissuti emozionali ai tempi del COVID-19: una ricerca esplorativa per riflettere sulle proprie esistenze. Lifelong Lifewide Learning, 16(35), 372-398.

Castellví, J., Massip, M. y Pagès, J. (2019). Emociones y pensamiento crítico en la era digital: un estudio con alumnado de formación inicial. REIDICS, 5, 23-41. https://doi.org/10.17398/2531-0968.05.23

Chetty, R., Grusky, D., Hendren, N., Hell, M., Manduca, R. y Narang, J. (2017). The Fading American Dream: Trends in Absolute Income Mobility Since 1940. Science, 356, 398-406.

CIS (2015). Indicador de confianza del consumidor. mes de diciembre de 2015. Estudio № 3122 . Recuperado de https://n9.cl/j18o

CIS (2016). Estudio no3134. Barómetro de abril 2016. Recuperado de http://www.cis.es/cis/opencm/ES/1_encuestas/estudios/ver.jsp?estudio=14277 
CIS (2020): Avance de resultados del estudio 3279. Barómetro especial de abril 2020. Recuperado de https://n9.cl/g8sag

Delval, J. y Denegri, M. (2002). Concepciones evolutivas acerca de la fabricación del dinero. I. Investigación en la escuela, 48, 39-54.

Delval, J. y Kohen, R. (2010). La crisis en la escuela. Cuadernos de Pedagogía, 405, 44-50.

Donat, M. y Martín-Lagos, M. (2020). Jóvenes boomerang en Andalucía: quiénes son y por qué regresan. Revista Española De Sociología, 29(3-1), 39-56. https://doi.org/10.22325/fes/res.2020.56

Du Bois-Reymond, M., y López Blasco, A. (2004). Transiciones tipo yo-yo y trayectorias fallidas: hacia las políticas integradas de transición para los jóvenes europeos. Revista de Estudios de Juventud, 65(4), 11-29.

Estefanía, J. (2017). Abuelo, ¿cómo habéis consentido esto? Barcelona: Planeta.

Eurofound (2012). Third European Quality of Life Survey - Quality of life in Europe: Impacts of the crisis. Luxemburgo: Oficina Unión Europea.

Eurofound (2014). Social situation of young people in Europe. Luxemburgo: Oficina Unión Europea. Eurofound (2020). Living, working and COVID-19, COVID-19 series. Luxemburgo: Oficina Unión Europea.

Freixa, C. y Nofre, J. (2013). \#Generaciónindignada. Barcelona: Milenio Editorial.

Freixa, C. y Rubio, C. (2017). Te vas pensando que has dejado atrás a zombis. La emigración juvenil: ¿aventura o exilio? Revista de Dialectología y Tradiciones Populares, 72(1), 9-22.

Furlong, A., Woodman, D. y Wyn, J. (2011). Changing Times, Changing Perspectives: Reconciling 'Transition' and 'Cultural' Perspectives on Youth and Young Adulthood. Journal of Sociology, 47(4), 355-370. https://doi.org/10.1177/1440783311420787

García-Gómez, S., Padilla Carmona, M.T. y Suárez Ortega, M. (2009). La imagen académica de alumnas de $4^{\circ}$ de E.S.O. según su profesorado, sus familias y las propias chicas. En A. Boza, J.M. Méndez, M. Monescillos, M. Toscano, M.Cinta, J.A. Ávila, J. Tello y M. Salas (coord.), XIV Congreso Nacional de modelos de Investigación Educativa. Educación, investigación y desarrollo social (pp. 1385-1392). Universidad de Huelva/AIDIPE: Huelva

Gil-Hernández, C., Bernardi, F. y Luijkx, R. (2020). Intergenerational Social Mobility in TwentiethCentury Spain: Social Fluidity without Educational Equalization? En Richard Breen y Walter Müller (ed.), Education and Intergenerational Social Mobility in Europe and the United States (pp. 224-250). Stanford University Press: California.

Heckhausen J., Wrosch, C. y Schulz, R. (2010). A motivational theory of life-span development. Psychological Review, 117(1), 32-60.

INJUVE (2012). Jóvenes, Actitudes Sociales y Políticas y Movimiento 15-M. Recuperado de http://www.injuve.es/sites/default/files/2012/28/publicaciones/2Sondeo\%202011.2b. pdf

INJUVE (2020). Juventud en riesgo: análisis de las consecuencias socioeconómicas de la COVID-19 sobre la población joven en España. Informe de urgencia (junio-julio). Recuperado de http://www.injuve.es/sites/default/files/adjuntos/2020/10/juventudenriesgo_informe2. pdf 
Morán, C. (20 de marzo de 2012). ¿Generación cangrejo? No parece. El País. Recuperado de https://elpais.com/politica/2012/03/20/nimileurista/1332270873_862619.html

OCDE (2015). Girls' lack of self-confidence. En OECD (Ed.), The ABC of Gender Equality in Education: Aptitude, Behaviour, Confidence (pp. 63-95). París: OECD Publishing.

OIT (2020). Los jóvenes y la COVID-19. Efectos en los empleos, la educación, los derechos y el bienestar mental. Disponible en https://www.ilo.org/global/topics/youthemployment/publications/WCMS_753054/lang--es/index.htm

Olmos, R. (2016). La recepción de la propaganda política en adolescentes durante un contexto de crisis. Revista Prisma Social, 17, 162-183.

Olmos, R. (2018a). La crisis económica en el aula: las representaciones sociales de un grupo de chicos y chicas en el tiempo y sus perspectivas de futuro. Enseñanza de las ciencias sociales: revista de investigación, 17, 23-35.

Olmos, R. (2018b). Las narrativas de un grupo de chicos y chicas adolescentes sobre la crisis económica. En E. López, C. R. García y M. Sánchez (Eds.), Buscando formas de enseñar: investigar para innovar en Didáctica de las Ciencias Sociales (pp. 1017-1026). Valladolid: Ediciones Universidad de Valladolid y AUPDCS.

Olmos, R. (2020). ¿Es capaz el alumnado de discernir en la infodemia del coronavirus? La influencia de las noticias falsas en sus relatos. Revista De Estilos De Aprendizaje, 13(26), 73 84.

Olmos, R., González-Monfort, N. y Pagès, J. (2017). Las representaciones sociales del alumnado sobre la crisis. ¿Qué soluciones ofrece el alumnado ante los problemas económicos? Didáctica de las Ciencias Experimentales y Sociales, 32, 51-70.

Olmos, R. y Pagès, J. (2017). Cambios y continuidades en las representaciones sociales de un mismo grupo de chicos y chicas sobre la crisis económica. En R. Martínez, R. García-Morís y C.R. García (Eds.), Investigación en didáctica de las ciencias sociales. Retos, preguntas y líneas de investigación, (pp. 720-729). Córdoba: Universidad de Córdoba y AUPDCS.

Olmos, R. y Pagès, J. (2018). Quines són les aspiracions socioprofessionals i els projectes de vida dels nois i noies en un context de crisi económica? En B. Tossar, A. Santisteban y J.Pagès (Coords.), Què està passant al món? Què estem ensenyant? Per un ensenyament de les ciències socials centrat en els problemes, la justicia social i la ciutadania global (pp. 325-334). Barcelona: Servei de Publicacions de la UAB.

Padilla, M.T., García-Gómez, M.S. y Suárez, M. (2010). Diferencias de género en el autoconcepto general y académico de estudiantes de 4을 de ESO. Revista de Educación, 352, 495-515.

Pagès, J. y González-Monfort, N. (2010). ¿Por qué y qué enseñar sobre la crisis? Cuadernos de Pedagogía, 405, 52-60.

Pagès, J. (2015). La educación política y la enseñanza de la actualidad en una sociedad democrática. Educao em foco. Consciencia do Mundo Histórico Social: caminhos investigativos, 19(3), 17-35.

Pagès, J. (2019). Ciudadanía global y enseñanza de las Ciencias Sociales: retos y posibilidades para el futuro. REIDICS, 5, 5-22. https://doi.org/10.17398/2531-0968.05.5

Politikon (2017). El muro invisible. Las dificultades de ser joven en España. Barcelona: Debate. 
Rudolph, C. W. y Zacher, H. (2020). COVID-19 and careers: On the futility of generational $\begin{array}{llll}\text { explanations. Journal of } & \text { Becational }\end{array}$ https://doi.org/10.1016/j.jvb.2020.103433

Sánchez-Galán, F.J. (2020). Los eventos de transición hacia la adultez en España. La influencia de los factores que la determinan. Sociología y tecnociencia: Revista digital de sociología del sistema tecnocientífico, 10(1), 159-179.

Sánchez-Rojo, A. y Prieto, M. (2020). Jóvenes transitando a la educación posobligatoria: el reflejo de la injusticia hermenéutica en sus discursos. Revista de Sociología de la Educación-RASE, 13(4), 579-596.

Sandel, M.J. (2020). La tiranía del mérito: ¿Qué ha sido del bien común? Barcelona: Debate.

Sanmartín, A., Ballesteros, J.C., Calderón, D. y Kuric, S. (2020). De puertas adentro y de pantallas afuera. Jóvenes en confinamiento. Madrid: Centro Reina Sofía sobre Adolescencia y Juventud y FAD. http://doi.org/10.5281/zenodo.4054836

Schwarz, G. (2020). American Dream 2020 snapshot: How americans views the American dream and economic mobility. Recuperado de https://www.archbridgeinstitute.org/2020/10/27/american-dream-2020-snapshot/

Serrano, L., Soler, A. y Pascual, F. (2020). Vulnerabilidad a corto plazo del empleo ante el Coronavirus: España y la Comunitat Valenciana. COVID 19: IVIE express. Instituto Valenciano de Investigaciones Económicas.

Tarabini, A., Curran, M., Castejón, A. y Montes, A. (2018). Framing youth educational choices at the end of compulsory schooling: the Catalan case. En A. Tarabini y N. Ingram, (Eds.), Educational Choices, Transitions and Aspirations in Europe (pp. 107-121). Londres: Routledge.

UNESCO (2019). Descifrar el código: la educación de las niñas y las mujeres en ciencias, tecnología, ingeniería y matemáticas (STEM). París: UNESCO. Recuperado de https://unesdoc.unesco.org/ark:/48223/pf0000366649

Vallejo, A. (2017). La generación Y ante el desafío de su inserción laboral: realidades frente a estereotipos. Arbor, 193 (783), a375. http://dx.doi.org/10.3989/arbor.2017.783n1006

Verdugo, L., Freire, T. y Sánchez-Sandoval, Y. (2018). Las relaciones entre autopercepciones y expectativas futuras: un estudio con preadolescentes españoles y portugueses. Revista de Psicodidáctica, 23(1), 39-47. https://doi.org/10.1016/j.psicoe.2017.07.001

Zurdo, Á. y López de la Nieta, M. (2013). Estrategias e imágenes sobre la crisis en el espacio social de la nueva pobreza. Representaciones sociales y atribuciones causales. Cuadernos de Relaciones $\quad$ 31(2), 383-433. http://dx.doi.org/10.5209/rev_CRLA.2013.v31.n2.43224 